\title{
Noise Reduction by Applying Modulation Principles
}

\author{
Donald Ewat.d, Armin Paviovic, and John G. Bollinger \\ The University of Wisconsin, Department of Mechanical Engineering, Madison, Wisconsin 53705
}

\begin{abstract}
This paper presents techniques for determining nonperiodic spacing of events for the purpose of reshaping noise-frequency spectra. The object of the application is to provide reduced noise levels and the redistribution of the frequencies at which there is noise energy so as to generate fewer perceptible sounds. Three analytical techniques for predicting the noise spectrum resulting from nonuniform spacing are presented. Emphasis is focused on a semigraphical design technique using Bessel functions, which has proven to be of great value in the actual selection of modulated event spacing. Fourier analysis of an impulse approximation and a sinusoidal wave approximation are two other alternative techniques presented. The techniques outlined are applied to the problem of reshaping the noise spectrum of a 22 -blade fan in a 5-hp induction motor. A comparison of predicted results provides an evaluation of the alternative approaches.
\end{abstract}

\section{INTRODUCTION}

A problem of noise reduction in machinery generally involves the reduction, shifting, or redistribution of pure-tone effects which result from cyclic events in the operation of the equipment.

K. D. Kryter and K. S. Pearsons ${ }^{1}$ showed that bandlimited random noise is perceived to be less noisy than sound composed of pure-tone superimposed on bandlimited random noise, if the over-all sound-pressure levels (SPLs) are the same. They also showed that sounds of larger tone-to-noise ratios are judged to be noisier, for equal SPL.

It might be concluded that sound consisting of a pure tone superimposed on random background noise can be made to sound less noisy by dispersing the energy of the tone over a number of discrete frequencies. Thus, a system design should aim to minimize pure-tone effects as well as to reduce the over-all broad-band noise level.

This paper is primarily concerned with presenting analytical methods that may be used to determine event spacing to control the spectral distribution of pure tones in the over-all noise spectrum generated by the equipment components.

As an example of an approach to reduction of noise generation, this paper will consider the air-cooling system in a 5 -hp induction motor. In the over-all analysis, it is necessary to include a careful study of fan characteristics and temperature distribution as well as air-path geometry and over-all system cost. A boundary condition might be that any design must not allow the over-all winding temperature rise to exceed a specified limit.

This problem may be approached by using the following design procedures to minimize the air noise in two broad areas:

(1) Optimize the air-flow path configuration to maximize the cooling effect of the air.

(2) Select the number of blades to give maximum air flow for a given fan diameter.

(3) Reduce the diameter of the fan as much as possible to reduce the tip velocity.

(4) Space the fan blades unevenly to control puretone effects.

To implement the control of pure-tone effects described in procedure 4, several analytical approaches will be demonstrated.

The centrifugal fan is a device which produces a number of disturbances, or events, per operating cycle. Its cycle is one-shaft revolution. It produces a pressure disturbance for each blade which passes a fixed reference point. The fundamental frequency for the disturbances is the blade passing frequency, i.e., the product of shaft rotational frequency and number of blades.

A straightforward approach to selecting the nonuniform spacing is to choose a modulation principle. For example, the events may be sinusoidally phase modulated geometrically. The result in the noise spectrum is to generate side bands of a fundamental pure tone, thereby reducing the magnitude of the funda- 


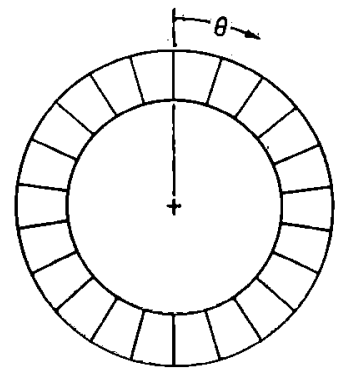

(a)

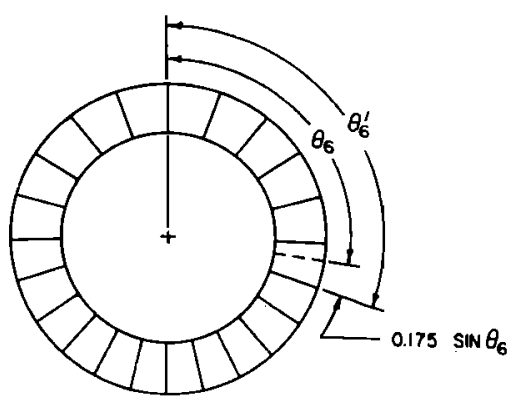

(b)

FIG. 1. Sinusoidal modulation of a 22-blade fan. (a) Evenly spaced fan. (b) Modulated fan.

mental. The resulting tones, if properly reduced in amplitude and located in the proper bandwidth, may be buried in the background noise.

Sinusoidal modulation of a fan-blade spacing involves the modification of the position of equally spaced blades about their nominal positions in a sinusoidal amplitude pattern.

Modulated positions are described by:

$$
\theta_{i}{ }^{\prime}=\theta_{i}+\Delta \theta \sin \left(m \theta_{i}\right)
$$

where $\theta_{i}$ is the $i$ th blade position in an evenly spaced fan arrangement, $\theta_{i}{ }^{\prime}$ is the $i$ th blade position after rearranging the blades, $\Delta \theta$ is some maximum bladeangle change (the modulation amplitude), and $m$ is the number of times the modulation cycle is repeated in one revolution of the fan.

Figure 1 shows the blade spacing of a 22-blade fan before and after it was modulated by angular spacing of the form

$$
\theta_{i}{ }^{\prime}=\theta_{i}+(0.175 \mathrm{rad}) \cdot \sin \left(\theta_{i}\right) \text {. }
$$

Figure 1(a) shows the angular locations of evenly spaced blades before modulation. Figure 1(b) shows how the blades are spaced after sinusoidal modulation according to Eq. 2.

The noise resulting from sinusoidal modulation of the fundamental blade passing tone may be expressed by the classical sinusoidal phase-modulation equation

$$
f(t)=A_{0} \sin \left(2 \pi F_{0} t+\Delta \phi \sin 2 \pi \nu t\right),
$$

where $A_{0}$ is amplitude of the fundamental blade passing tone; $F_{0}=I f_{s}$, blade passing frequency; $I$, number of blades; $f_{s}$, shaft rotational frequency; $\nu=m f_{s}$, the modulation frequency; and $\Delta \phi=I \Delta \theta$, phase-modulation amplitude.

$\Delta \phi$ refers to an angle which goes from zero to $2 \pi$ during each nominal blade spacing, and $\Delta \theta$ is an angle which goes from zero to $2 \pi$ for each revolution of the shaft. This means that $\Delta \phi$ will go from zero to $2 \pi I$ times for every time that $\Delta \theta$ goes from zero to $2 \pi$.

In the case of the fan in Fig. 1(b), this modulation cycle repeats itself once $(m=1)$ for each cycle of the fan.

By using the trigonometric relations

$$
\begin{aligned}
\sin p \cos q & =\frac{1}{2}[\sin (p+q)+\sin (p-q)], \\
\sin (p+q) & =\sin p \cos q+\cos p \sin q
\end{aligned}
$$

and the relations between the Bessel and trigonometric functions

$$
\begin{aligned}
& \cos (p \sin q)=J_{0}(p)+2 \sum_{n=1}^{\infty}\left[J_{2 n}(p) \cos (2 n q)\right], \\
& \sin (p \sin q)=2 \sum_{n=1}^{\infty}\left[J_{2 n-1}(p) \sin (2 n-1) q\right],
\end{aligned}
$$

where $J_{n}(p)$ is the Bessel function of the first kind, order $n$, argument $p$, it can be shown that

$$
\begin{aligned}
f(l)= & A_{0}\left\{J_{0}(\Delta \phi) \sin \left(2 \pi F_{0} t\right)\right. \\
& +\sum_{n=1}^{\infty} J_{n}(\Delta \phi) \sin \left[2 \pi\left(F_{0}+n \nu\right) t\right] \\
& \left.\quad+\sum_{n=1}^{\infty}(-1)^{n} J_{n}(\Delta \phi) \sin \left[2 \pi\left(F_{0}-n \nu\right) t\right]\right\}
\end{aligned}
$$

Equation 4 illustrates that the frequency spectrum will consist of a center frequency at $F_{0}$ with an amplitude of $A_{0} J_{0}(\Delta \phi)$ and a number of side bands at integer multiples of $\nu$ from the center frequency, with amplitudes symmetric about the center frequency.

The values of $J_{n}(\Delta \phi)$ may be found in many mathematical handbooks and are shown graphically in Fig. 2(a).

An example of how the frequency spectrum may be determined for a given $\Delta \phi$ is shown in Fig. 2.

A trial value is chosen for $\Delta \phi$. Then a vertical line is drawn through the trial value of $\Delta \phi$. The intersection of this line with the $J_{n}(\Delta \phi)$ curves indicates the relative amplitudes of the resulting components at frequencies $F_{0} \pm n \nu$. The resulting frequency spectrum, Fig. 2(b), is given to the right of the graph. Note that absolute values are plotted on the frequency spectrum shown in Fig. 2(b). The dashed lines in Fig. 2(b) indicate the normalized amplitude of the fundamental blade passing frequency tone for a fan with evenly spaced blades.

The example in Fig. 2 is for the modulated blade spacing shown in Fig. 1(b). In this example, $I=22$, $m=1, f_{s}=30 \mathrm{~Hz}, \nu=30 \mathrm{~Hz}$, and $F_{0}=660 \mathrm{~Hz}$. 
Fig. 2. (a) Bessel coefficients versus maximum phase deviation $(\Delta \phi)$. (b) Frequency spectrum for the phase-modulated fan with $\Delta \phi=3.85$.

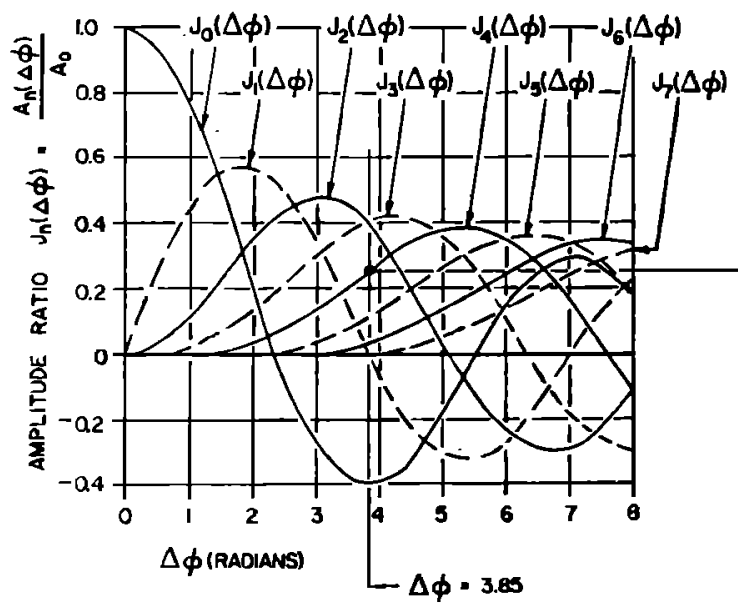

(a)

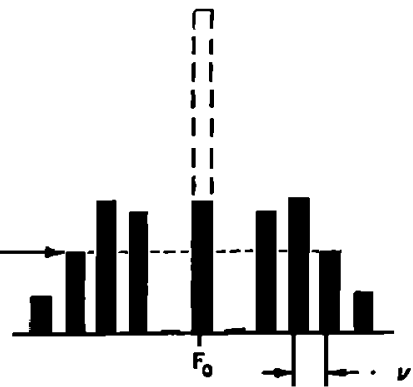

(b)
The Bessel series is for a continuous phase-modulated function, while the actual frequency spectrum of the fan is produced by a number of more nearly discrete events. The amplitudes in the frequency spectrum obtained from the Bessel series will therefore differ somewhat from those obtained from the fan. The Bessel series, however, will more closely approximate the actual fan spectrum when the number of blades is large.

For fans which have a small number of blades, a more realistic result may be estimated by Fourier analysis of the pressure waveform produced by the fan blades.

\section{FOURIER ANALYSIS}

The Fourier series analysis assumes that the function is periodic over one revolution of the fan or one cycle of a group of events. The function is represented by an infinite orthogonal trigonometric series such as

$$
f(t)=\bar{f}(l)+\sum_{n=1}^{\infty} B_{n} \sin (n \omega t)+\sum_{n=1}^{\infty} C_{n} \cos (n \omega t),
$$

where

and

$$
B_{n}=\frac{\omega}{\pi} \int_{0}^{2 \pi / \omega} f(t) \sin (n \omega t) d t
$$

$$
C_{n}=\frac{\omega}{\pi} \int_{0}^{2 \pi / \omega} f(t) \cos (n \omega t) d l .
$$

Since the fan traveling at angular velocity $\omega=2 \pi f$ 。 will be analyzed in terms of the angular positions of the blades, $B_{n}$ and $C_{n}$ can be written in terms of the angular position of the fan, $\theta$, by using the substitutions $\theta=\omega t$ and $d \theta=\omega d t$ in Eqs. 6 and 7 to yield

and

$$
B_{n}=\frac{1}{\pi} \int_{0}^{2 \pi} f(\theta) \sin (n \theta) d \theta
$$

$$
C_{n}=\frac{1}{\pi} \int_{0}^{2 \pi} f(\theta) \cos (n \theta) d \theta
$$

Since the phase angle of the harmonic components is generally not of interest in noise analysis, only the amplitude, $A_{n}=\left(B_{n}^{2}+C_{n}{ }^{2}\right)^{3}$, needs to be calculated.

A Fourier analysis of the noise generated for modified blade spacing requires some knowledge of the waveform, which in turn depends on the position of the blades. This waveform may be approximated by an arbitrarily chosen function. Two such functions are impulses occurring at blade tip locations and singleperiod sinusoids whose periods are equivalent to the blade_spacings.

\section{IMPULSE}

The easiest waveform to analyze would result from the assumption that an impulse of strength $K, K \delta(\theta)$ is produced by each blade. In this case, the function $f(\theta)$ becomes

$$
\begin{aligned}
f(\theta) & =K \delta(\theta), \text { for } \theta=\theta_{i}, \quad i=1, \cdots, I, \\
& =0, \text { for all other } \theta,
\end{aligned}
$$

where $\theta_{i}$ is the angular position of the $i$ th blade, and $I$ is the number of blades in the fan.

Since $\int K \delta(\theta) d \theta=K$, substituting this function for $f(\theta)$ into the equation for $B_{n}$ and $C_{n}$ yields

and

$$
\begin{aligned}
& B_{n}=\frac{K}{\pi} \sum_{i=1}^{T} \sin \left(n \theta_{i}\right) \\
& C_{n}=\frac{K}{\pi} \sum_{i=1}^{I} \cos \left(n \theta_{i}\right) .
\end{aligned}
$$

Letting $K=\pi / I$, these equations yield

$$
A_{I}=A_{2 I}=A_{3 I}=\cdots=1.0
$$

for an evenly spaced fan arrangement, where $A_{n I}$ is the amplitude at frequency $n f_{a} I$.

The main problem with the assumption of an impulse waveform in this case is evidenced above. All harmonics 
EWALD, PAVLOVIC, AND BOLLINGER

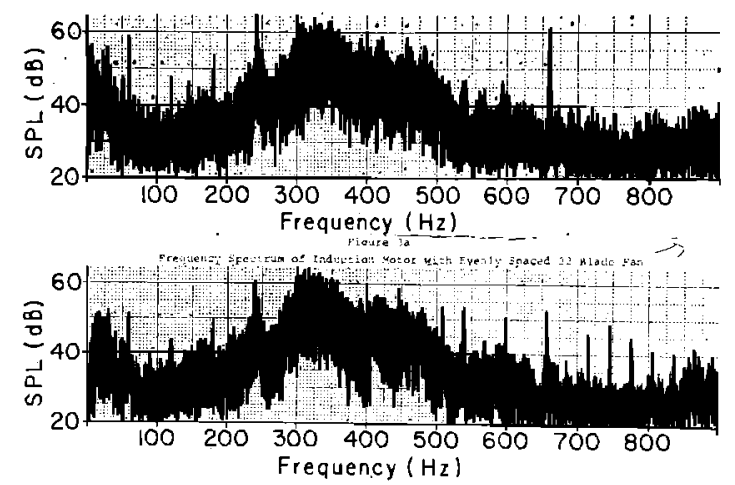

Fig. 3. (a) Frequency spectrum of an induction motor with an evenly spaced 22-blade fan. (b) Frequency spectrum of an induction motor with blade spacing of Fig. $1(\mathrm{~b})$.

are equal in amplitude. An examination of an actual frequency spectrum for an evenly spaced fan arrangement shows that this is not true. The higher harmonics in reality have smaller amplitudes.

\section{SINUSOIDAL APPROXIMATION}

In the case of unequally spaced blades, one approach to the analysis is to assume single-period sine terms with period equal to the blade spacing. The equation for this function is

$$
f(\theta)=\sum_{i=1}^{I} \sin \frac{2 \pi\left(\theta-\theta_{i}\right)}{\theta_{i+1}-\theta_{i}}, \text { for } \theta_{i}<\theta<\theta_{i+1},
$$

where $\theta_{I+1}=\theta_{1}+2 \pi \mathrm{rad}$. Letting $D_{i}=2 \pi /\left(\theta_{i+1}-\theta_{i}\right)$ and substituting into the equations for $B_{n}$ and $C_{n}$ yields

$B_{n}=\frac{1}{2 \pi} \sum_{i=1}^{I}\left\{\frac{\sin \left[\left(D_{i}-n\right) \theta-D_{i} \theta_{i}\right]}{D_{i}-n}\right.$

$$
\left.-\frac{\sin \left[\left(D_{i}+n\right) \theta-D_{i} \theta_{i}\right]}{D_{i}+n}\right\}_{\theta_{i}}^{\theta_{i+1}}
$$

and

$$
\begin{aligned}
& C_{n}=\frac{-1}{2 \pi} \sum_{i=1}^{I}\left\{\frac{\cos \left[\left(D_{i}+n\right) \theta-D_{i} \theta_{i}\right]}{D_{i}+n}\right. \\
&\left.-\frac{\cos \left[\left(D_{i}-n\right) \theta-D_{i} \theta_{i}\right]}{D_{i}-n}\right\}_{\theta_{i}}^{\theta_{i+1}} .
\end{aligned}
$$

The values of $B_{n}$ and $C_{n}$ are defined for all $n \neq D_{i}$. The values of the functions at $\left(\theta_{i+1}-\theta_{i}\right)=2 \pi / n$ must be evaluated by use of L'Hospital's rule. Taking the derivative of both the numerator and the denominator with respect to $D_{i}$ and evaluating them at $D_{i}=n$ yields

$$
\begin{array}{r}
\lim _{D_{i} \rightarrow n}\left\{\frac{\sin \left[\left(D_{i}-n\right) \theta_{i+1}-D_{i} \theta_{i}\right]-\sin \left[\left(D_{i}-n\right) \theta_{i}-D_{i} \theta_{i}\right]}{\left(D_{i}-n\right)}\right\} \\
=\left(\theta_{i+1}-\theta_{i}\right) \cos \left(D_{i} \theta_{i}\right),
\end{array}
$$

and

$$
\begin{array}{r}
\lim _{D_{i} \rightarrow n}\left\{\frac{\cos \left[\left(D_{i}-n\right) \theta_{i+1}-D_{i} \theta_{i}\right]-\cos \left[\left(D_{i}-n\right) \theta_{i}-D_{i} \theta_{i}\right]}{\left(D_{i}-n\right)}\right\} \\
=\left(\theta_{i+1}-\theta_{i}\right) \sin \left(D_{i} \theta_{i}\right) .
\end{array}
$$

Use of the sinusoidal waveform results in more complicated calculations than the impulse waveform. However, it produces more realistic results in that the harmonic content will more closely resemble that of the hardware. This is especially important when phase modulation causes the side bands of successive harmonics to overlap in the frequency spectrum.

\section{EXPERIMENTAL RESULTS}

These methods for analyzing and predicting a frequency spectrum were used to determine a fan arrangement for a 5-hp induction motor having a shaft rotational frequency of $30 \mathrm{~Hz}$.

The original fan on the motor had 22 evenly spaced blades and therefore the fundamental disturbance frequency, $F_{0}$, was $660 \mathrm{~Hz}$. This number of blades was retained for the modulated arrangement because the $660-\mathrm{Hz}$ blade passing frequency was in an area of relatively low background-noise level [see Fig. 3(a)]. The band of low-level background noise extends about $150 \mathrm{~Hz}$ below the blade passing frequency to allow five significant side-band pairs to be produced in a fan which has one modulation cycle for each revolution of the fan $(m=1)$.

The modulation amplitude, $\Delta \theta$, of $0.175 \mathrm{rad}$ was chosen to ensure low amplitudes at all frequencies while limiting the number of significant side-band pairs to four. Note that $\Delta \phi=\Delta \theta \times I=0.175 \times 22=3.85 \mathrm{rad}$, indicated on Fig. 2(a). The half bandwidth in which significant side-band amplitudes occur ${ }^{2}$ is $\frac{1}{2} B W=\nu \Delta \phi$ $=m \times f_{s} \times \Delta \phi=1 \times 30 \times 3.85 \simeq 115 \mathrm{~Hz}$.

\section{COMPARISON}

The frequency spectrum of the induction motor with evenly spaced blades is shown in Fig. 3(a). The spectrum for the modulated 22-blade fan is shown in Fig. 3 (b). The amplitudes which are evident above the background level in this spectrum are shown in Fig. 4(d). Figs. 4(a), 4(b), and 4(c) show the frequency spectra predicted using the methods of analysis discussed earlier in this paper.

All three methods produced good estimates of the frequency spectrum which would be produced by the modulated fan. This fact is explained by the large number of blades and the small blade-angle changes involved in the modulated fan. The large number of blades made the system approximate a continuous system. The small degree of modulation prevented the higher harmonics in the Fourier analysis of the impulse train from appearing in the low-frequency ranges. The 
higher harmonics were ignored, since it was known that their amplitudes were insignificant.

The Bessel series analysis is useful because it permits the designer to tailor the frequency spectrum by direct visual inspection of Fig. 2. This analysis is best for large numbers of blades and small modulation amplitudes.

The Fourier analysis of an impulse for each blade gives good results in the special cases of small modulation amplitudes (a) where side bands from the higher harmonics do not overlap with those of the fundamental, or (b) where the higher harmonics of the fan are nearly equal in amplitude to the fundamental frequency.

The Fourier analysis of a sine wave between blades requires more calculations, but the predicted spectrum is the most accurate when the fundamental frequency is much greater in amplitude than the higher harmonics.

\section{vI. CONCLUSION}

Nonuniform event spacing in multi-event cyclic processes results in reduction of the amplitudes of tonal noise components. In the illustrative example, sinusoidal phase modulation resulted in a series of tones with amplitudes at least $8 \mathrm{~dB}$ below that of the unmodulated tone, while reasonable blade spacing was maintained. The use of Fig. 2 permits rapid and convenient selection of event spacing. In cases where the number of events per cycle is small (say 20 or less), Fourier analysis may be done, using assumed waveforms, as a check on the predictions of Fig. 2.

In the application of the induction motor fan cited, the $660-\mathrm{Hz}$ blade passing tone was distinctly perceptible in the original design. Following modification of the fan by application of the principles of phase modulation discussed in this paper, the $660-\mathrm{Hz}$ blade passing tone became audibly imperceptible. The one-fold modulation $(m=1)$ chosen for this application did not result in an undesirable mechanical unbalance or aerodynamic loss. However, in other applications, this aspect should be critically evaluated. It is interesting to note that the mechanical balance, after modulation, is perfect in all cases where $m$ is an integer greater than 1 and where the number of blades, $I$, is an integer multiple of $m$. The frequency spectrum in Fig. 3(b) may be further (a)

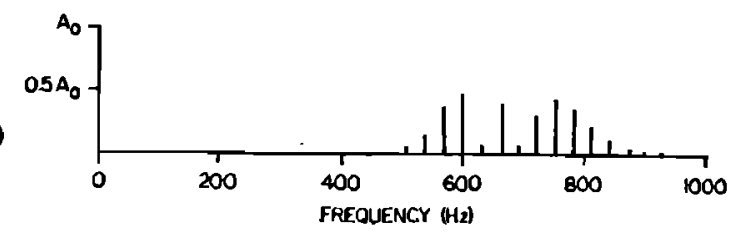

(b)

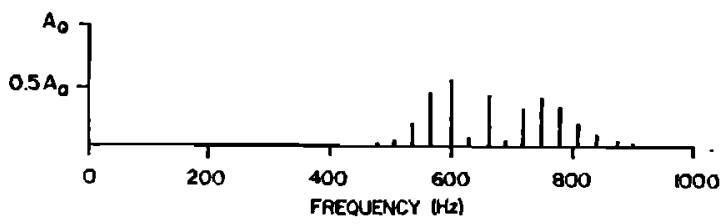

(c)

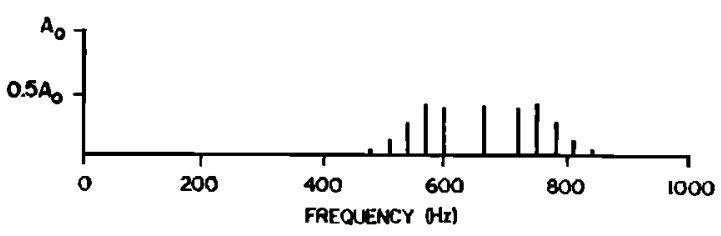

(d)

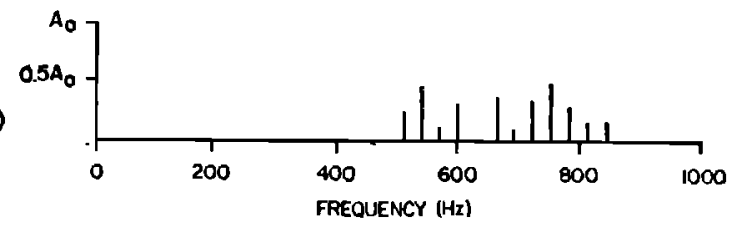

FIG. 4. Frequency spectra for the sinusoidally modulated 22-blade fan shown in Fig. 3. (a) Spectrum predicted using Fourier analysis of an impulse for each blade. (b) Spectrum predicted using Fourier analysis of a sinusoidal waveform between blades. (c) Spectrum predicted using Bessel series coefficients. (d) Actual spectrum shown in Fig. 3(b), amplitudes measured above background noise.

altered by other geometric modification of the fan, such as the reduction of fan diameter. The result effected is the further burying of the tonal noise in the broad-band spectrum.

1 K. D. Kryter and K. S. Pearsons, "Judged Noisiness of a Band of Random Noise Containing an Audible Pure Tone," J. Acoust. Soc. Amer. 38, 106-112 (1965).

'S. Goldman, Frequency Analysis, Modulation and Noise (Dover, New York, 1948). 